\title{
Serum sialic acid could be a diagnostic candidate of unstable angina and its expression in different numbers of lesion vessels in acute myocardial infarction: a retrospective study
}

\author{
Yuling Wu \\ hospital affiliated of Qingdao University https://orcid.org/0000-0001-9962-6211 \\ Nana Pan \\ Affiliated hospital of Qingdao University
}

Lijuan Tan ( $\nabla$ qdwyl2020@126.com)

Affiliated hospital of Qingdao University https://orcid.org/0000-0002-2847-1352

\section{Research article}

Keywords: Serum sialic acid, acute coronary syndrome, diagnosis, prognosis, lesion vessels

Posted Date: September 8th, 2020

DOI: https://doi.org/10.21203/rs.3.rs-65498/v1

License: (c) (i) This work is licensed under a Creative Commons Attribution 4.0 International License. Read Full License 


\section{Abstract}

Background: Acute coronary syndrome is one of the leading causes of morbidity and mortality worldwide. Intracoronary thrombosis caused by atherosclerotic unstable plaque rupture or erosion is considered as main pathological basis. There have no identified biomarkers about diagnosis of unstable angina and prognosis of acute myocardial infarction.

Methods: A total of 509 consecutive patients admitted to the Affiliated Hospital of Qingdao University from February 2019 to December 2019 were successively recruited in this retrospective study and divided into three groups, unstable angina, acute myocardial infarction and death occurred within 24 hours of acute myocardial infarction in hospital and then to compare with a total of 87 healthy people (control group) during the same time from Regular Physical Examination Center of the Affiliated Hospital of Qingdao University. Mann-Whitney test, Kruskal-Wallis test, and Chisquared test were used to analyze different types of grouping. Univariate and multivariate regression analyses were performed to analyze the risk factors. Relative operating characteristic (ROC) curve analysis was also performed to determine the power of test.

Results: our study suggested that serum sialic acid has a good diagnostic value in patients with unstable angina. Moreover, serum sialic acid is an independent risk factor of acute myocardial infarction. Serum sialic acid could be a candidate to distinguish Single/Double vascular lesions from Triple vascular lesions in acute myocardial infarction.

Conclusions: serum sialic acid is a potential biomarker to distinguish unstable angina from normal and estimate the number of diseased vessels in patients with acute myocardial infarction.

\section{Background}

Acute coronary syndrome (ACS) is a group of syndromes caused by acute myocardial ischemia, of which leading system is chest pain. Based on the electrocardiogram, suspected patients can be divided into one is termed STelevation ACS and another is non-ST-elevation ACS[1]. The former is interpreted as ST segment elevation myocardial infarction (STEMI) and the later includes unstable angina (UA) and non-ST segment elevation myocardial infarction (NSTEMI). Currently, atherothrombosis resulted from the disruption (rupture/erosion) of unstable plaque is considered as main pathological basis of ACS.

Presence of high sensitivity cardiac troponin (hs-cTn) greatly improves diagnosis of myocardial infarction and reduces mortality[2]. However, elevated cTn is not only in patients with MI, but associated with disease such as sepsis, heart failure and so on[3]. In addition, there are no identified indicators to diagnose UA and evaluate prognosis of MI. Therefore, additional biomarkers need to be found.

Sialic acid (SA) is a derivate of nine-carbon monosaccharides and exists in the terminal of the oligosaccharide chains of many glycoproteins and glycolipids, which play significant roles in physiological and pathological processes, such as cancer metastasis, viral infection and so on[4]. Many studies confirm that SA is elevated in cardiovascular disease and patients with AMI or UA have much higher SA level than those with stable angina or normal person[5]. Acting as a signaling molecule, a member of sialic acid, N-Acetyl-Neuraminic Acid (Neu5Ac) can trigger myocardial injury by activating Rho/ROCK-JNK/ERK signaling pathway in AMI mouse model, which provides evidence to clarify molecular mechanism of SA involved in coronary artery disease[6]. In this study, we aim to further verify the distributional difference of SA among subgroups of ACS and association between SA and prognosis of AMI, so as to assist in diagnosis and prognosis of ACS. 


\section{Methods}

\section{Study population}

A total of 509 consecutive patients admitted to the Affiliated Hospital of Qingdao University from February 2019 to December 2019 were successively recruited in this retrospective study and divided into three groups, UA, AMI and death occurred within 24 hours of AMI in hospital (called as Death in this article). All patients met the diagnosis criteria for ACS, which is based on the fourth global definition of myocardial infarction[7] and 2015 ESC Guidelines for the management of acute coronary syndromes[1]. In addition, we collected a total of 87 healthy people during the same time from Regular Physical Examination Center of the Affiliated Hospital of Qingdao University, which were identified by more than two clinicians.

Patients with cerebral infarction, sepsis, malignancy, severe renal and/or hepatic insufficiency, recent infection, previous history of cardiac diseases (AMI, severe valvular heart disease, and cardiomyopathy) were excluded.

\section{Clinical information}

The demographic data (age, gender, body mass index [BMI]), information on factors for ACS (smoking, alcoholism, blood pressure, blood glucose and blood lipid) and information about previous medications and severity of coronary lesions were collected after admission.

BMI was calculated by equation (weight $[\mathrm{kg}] /$ height squared $\left[\mathrm{m}^{2}\right]$ ). Smokers were person who smoked one and more cigarettes per day and drinking alcohol 50 gram per day was considered as alcoholism. Repeated blood pressure measurements (at least twice in different times) of systolic blood pressure $\geq 140 \mathrm{mmHg}$ and/or diastolic blood pressure $\geq 90 \mathrm{mmHg}$ or taking anti-hypertensive drugs were criteria for hypertension. Oral anti-diabetic agents/insulin or a fasting serum glucose level of $\geq 126 \mathrm{mg} / \mathrm{dL}(7.0 \mathrm{mmol} / \mathrm{L})$ were applicable to diagnose Diabetes. Fasting peripheral venous blood was collected in the morning of the second day after admission, and all data were obtained from the same blood sample. Cardiac function was classed in accordance with NYHA class in UA and Killip class in AMI. All recruited patients were accepted selective coronary angiography and the number of diseased vessels was recorded. Significant left main coronary artery lesion was considered as equivalent of triple vessel disease. Meanwhile, the severity of coronary artery was assessed according to Gensini Score[8]. What's mentioned above was identified by more than two experienced clinicians.

\section{Statistical analysis}

Statistical analyses were performed using SPSS software, version 22.0 and prism 6.0. The heat map was made by Excel 2016. Nonnormally distributed continuous variables are expressed as medians with interquartile range. Categorical variables are presented as counts and percentages. Mann-Whitney test was used to compare non-normally distributed continuous variables between the single/double group and triple group, whereas comparisons of nonnormally distributed continuous variables among control, UA, AMI, Death groups were performed with Kruskal-Wallis test. Categorical variables were compared using the Chisquared test. Univariate and multivariate regression analyses were performed to analyze the risk factors of UA and death occurred within 24 hours of AMI in hospital. Relative operating characteristic (ROC) curve analysis was also performed to determine the power of test of sialic acid for diagnosing UA and predicting death occurred within 24 hours of AMI in hospital. All P-values were two-tailed and it was considered statistically significant on the condition of $P<0.05$.

\section{Results}




\section{Baseline characteristics of the study population}

Except for death group, there were no statistical difference between the control group and the UA and AMI group on age, BMI, gender and alcoholism. There were statistical difference on systolic blood pressure, diastolic blood pressure and left ventricular ejection fraction. (Table 1). In addition, we graded cardiac function and recorded in Table 1. The laboratory parameters and Gensini Score (GS) of the four groups and their statistic differences were summarized in Table 2. To visualize data of laboratory parameters, we made a heat map based on Table 2 (Fig. 1).

\section{The expression of serum SA among patients with ACS}

SA increased with the severity of the disease(normal $<U A<A M I<$ Death) (Fig. 2). There were statistical significance between three experimental groups and control groups $\left(P_{1}<0.001, P_{2}<0.0001, P_{3}<0.0001\right.$ respectively). $P$ value among the experimental groups were $P_{U A}$ vs AMI $<0.0001, P_{U A}$ vs Death $<0.0001, P_{\text {AMI Vs Death }}<0.0001$. The relative operating characteristic curve (ROC curve) and their sensitivity, specificity and area under the curve (AUC) were presented in Fig. 3. SA as an indicator to differentiate UA and normal people had high specificity (89.66\%) yet low sensitivity (42.77\%) and AUC received 0.6815 (Fig. 3a). SA distinguished AMI from normal people with a sensitivity of $61.69 \%$ and a specificity of $97.7 \%$ (cut off value:560.4, AUC:0.8419, p<0.0001) (Fig. 3b). The cut off value of SA for predicting death occurred within 24 hours of AMI in hospital was $638.9 \mathrm{mg} / \mathrm{L}$ with a sensitivity of $66.67 \%$ and a specificity of $66.17 \%$ (AUC:0.7192, p < 0.0001) (Fig. 3f).

\section{The univariate and multivariate regression analysis on unstable angina}

Based on the Fig. 1, we placed some clinical parameters (SA, LDL, HDL, TG, LP(a), TC, Glu) who were statistical significance between UA group and control group into a enter univariate and multivariate logistic regression and result was recorded in Table 3. SA, TG were independent risk factors of UA (OR: 1.010, 95\%Cl: 1.005-1.015, P: 0.000; OR: 1.495, 95\%Cl: $1.084-2.060, \mathrm{P}: 0.014)$. HDL, LVEF were protective factors of UA (OR: $0.085,95 \% \mathrm{Cl}: 0.032-0.225, \mathrm{P}$ : 0.000; OR: $0.000,95 \% \mathrm{Cl}: 0.000-0.000, \mathrm{P}: 0.000)$. The multivariate regression analysis suggested that combination SA with HDL could improve capability of distinguishing UA from healthy people with a sensitivity of $64.94 \%$ and a specificity of $80.95 \%$ (AUC: $0.7754, \mathrm{P}<0.0001$ ). (Fig. 4)

\section{The univariate and multivariate regression analysis between AMI and Death}

Based on the Fig. 1, we placed some clinical parameters (BNP, SA, apoA1, Glu) and age, GS as well as LVEF who were statistical significance between AMI group and Death group into an enter univariate and multivariate logistic regression and results were recorded in Table 4. BNP, SA, Glu, GS and age were independent risk factors of Death (OR: 1.001, 95\%Cl: 1.001-1.002, P: 0.000; OR: 1.006, 95\%Cl: 1.002-1.010, P: 0.001; OR: 1.165, 95\%Cl: 1.005-1.349, P: 0.043; OR: 1.022, 95\%Cl: 1.008-1.036, P: 0.002; OR: 1.141, 95\%Cl: 1.071-1.215, P: 0.000). LVEF, apoA1 were protective factors of Death (OR: $0.048,95 \% \mathrm{Cl}: 0.004-0.512$, P: 0.012 ; OR: $0.000,95 \% \mathrm{Cl}: 0.000,0.018, \mathrm{P}: 0.000$ ). The multivariate regression analysis suggested that BNP and age were more significant independent risk factors than SA.

\section{The expression of serum SA in various number of lesion vessels in patients with AMI}

We divided patients with AMI into Single/Double vascular lesion group and Triple vascular lesion group based on record of coronary angiography. The baseline information was collected in Table 5. There were no statistical difference 
in gender, BMI, smoking, alcoholism, emergency, blood pressure, LVEF ( $P>0.05)$ while there had statistical difference in age and medical history of hypertension and diabetes $(P<0.05)$. The ROC of lesion vessels groups suggested that serum SA had a sensitivity of $63.51 \%$ and a specificity of $57.39 \%$ (AUC: $0.6005, P<0.05$ ) to differentiate Single/Double vascular lesions from Triple vascular lesions. (Fig. 5)

\section{Discussion}

Acute coronary syndrome (ACS) is one of the leading cause of morbidity and mortality worldwide. Intracoronary thrombosis caused by atherosclerotic unstable plaque rupture or erosion is considered as main pathological basis. Compared to acute myocardial infarction (AMI), the identification of biomarkers for the diagnosis, prognosis, and treatment of unstable angina (UA) remains a challenge in the clinic.[9] Although occurrence of cardiac troponin greatly improves accuracy of diagnosing $\mathrm{AMI}[10]$ and decreases mortality, there are no identification biomarkers to prognose AMI. The current study evaluates the diagnostic value of serum sialic acid in patients with UA and prognostic value of AMI. Sialic acid (SA) is the product of acetylated neuraminic acid and plays important roles in the physical activity of the body. SA is also involved in autoimmune disease[11], dementia[12], rheumatoid arthritis[13], chronic heart failure[14] and so on. SA is elevated in cardiovascular disease and patients with AMI or UA have much higher SA level than those with stable angina or normal person.[5] To better assist with diagnosis and prognosis of ACS, we tried to find relationship between serum SA and ACS.

Unstable angina (UA) is a very common complication in patients with coronary heart disease. $[15,16]$ The main clinical manifestation of UA is chest pain, pressure, or squeezing, which is caused by myocardial ischemia due to the obstruction or spasm of the coronary arteries. [17]There are several markers including C-reactive protein and interleukin-6[18], interleukin-10[19], interleukin-18[20], who have been studied to identified patients with UA. But those markers have their own limitations. Therefore, the putative markers of UA should still be explored.

Currently, diagnosis of acute myocardial infarction (AMI) is mainly completed by cardiac troponin. However, there are no identified biomarkers to be used to prognose development of AMI. In 1987, studies suggested that plasma sialidase activity increased in patients with acute myocardial infarction.[21] Zhang et al suggested that understanding of the molecular and cellular mechanisms about the SA metabolism might shed new light on curing atherosclerosis.[5] A study shows that serum total SA is at least in part related to some of the acute phase proteins in both healthy individuals and those having had a myocardial infarction.[22]

The concentration of serum lipid-bound sialic acid is related to the severity of coronary atherosclerosis, especially in patients with double/triple-vessel disease.[23]

In current study, serum SA has a good diagnostic value in patients with UA. Combination of SA and HDL greatly improves power of diagnosis. Moreover, serum SA is an independent risk factor of AMI. Serum SA could be a candidate to distinguish Single/Double vascular lesions from Triple vascular lesions in AMI.

\section{Conclusions}

The present study demonstrates that, in patients with ACS, serum SA is associated with UA and prognosis as well as numbers of lesion vessels in patients with AMI. The combination of serum SA and other traditional markers might be of great significance in identifying high-risk patients and providing timely intervention strategies to improve the diagnosis and prognosis of ACS.

\section{Declarations}


Ethics approval and consent to participate: This study was approved by Medical Ethics Committee of Affiliated Hospital of Qingdao University. This study was registered, number QYFY WZLL 25910.

Consent to publish: Not applicable

Availability of data and materials: The datasets used and/or analysed during the current study are available from the corresponding author on request.

Competing interests: The authors declare that they have no competing interests.

Funding: Not applicable

Authors' contributions: W YL participated in design of this study and drafted this manuscript. T LJ put up with this idea and designed this study. P NN assisted with clinical diagnosis and information collection. All authors read and approved the manuscript.

Acknowledgements: Not applicable

\section{Abbreviations}

ACS: Acute coronary syndrome; STEMI: ST segment elevation myocardial infarction; UA: unstable angina; NSTEMI: non-ST segment elevation myocardial infarction; hs-cTn: high sensitivity cardiac troponin; SA: Sialic acid; BMl: body mass index; SBP: systolic blood pressure; DBP: diastolic blood pressure; LVEF: left ventricular ejection fraction; AECI: angiotensin converting enzyme inhibitors; ARB: angiotensin receptor blocker; CCB: calcium channel blocker; cTnl: cardiac troponin l; CKMB: creatine kinase isoenzyme; BNP: type B natriuretic peptide; LDL: low density lipoprotein; TG: triglyceride; HDL: high density lipoprotein; FFA: free fatty acid; ApoA1: apolipoprotein A1; ApoB: apolipoprotein B; Lp(a): lipoprotein a; TC: total cholesterol; GLU: glucose; ADA: adenosine deaminase; AFU: fucosidase; LDH: lactic dehydrogenase; TP: total protein; A/G: albumin/globulin; GS: gensini score; ROC curve: the relative operating characteristic curve; AUC: area under the curve.

\section{References}

1. Roffi, M., et al., 2015 ESC Guidelines for the Management of Acute Coronary Syndromes in Patients Presenting Without Persistent ST-segment Elevation. Revista Espanola De Cardiologia, 2015. 68(12): p. 1125-1125.

2. Ladenson, J.H., A personal history of markers of myocyte injury [myocardial infarction]. Clinica Chimica Acta, 2007. 381(1): p. 3-8.

3. Keller, T., et al., Sensitive Troponin I Assay in Early Diagnosis of Acute Myocardial Infarction. New England Journal of Medicine, 2009. 361(9): p. 868-877.

4. Bull, C., et al., Sialic Acids Sweeten a Tumor's Life. Cancer Research, 2014. 74(12): p. 3199-3204.

5. Zhang, C., et al., Sialic acid metabolism as a potential therapeutic target of atherosclerosis. Lipids in Health and Disease, 2019. 18(1).

6. Zhang, L., et al., Functional Metabolomics Characterizes a Key Role for N-Acetylneuraminic Acid in Coronary Artery Diseases. Circulation, 2018. 137(13): p. 1374-+.

7. Thygesen, K., et al., Fourth Universal Definition of Myocardial Infarction (2018). Circulation, 2018. 138(20): p. E618-E651. 
8. Gensini, G.G., A more meaningful scoring system for determining the severity of coronary heart disease. Am J Cardiol, 1983. 51(3): p. 606.

9. Wang, J., et al., Identification of potential plasma biomarkers and metabolic dysfunction for unstable angina pectoris and its complication based on global metabolomics. Bioscience Reports, 2019. 39.

10. Park, K.C., et al., Cardiac troponins: from myocardial infarction to chronic disease. Cardiovasc Res, 2017. 113(14): p. 1708-1718.

11. Mahajan, V.S. and S. Pillai, Sialic acids and autoimmune disease. Immunol Rev, 2016. 269(1): p. $145-61$.

12. Davis, G., et al., Sialic acid, homocysteine and CRP: potential markers for dementia. Neurosci Lett, 2009. 465(3): p. 282-4.

13. Li, W., et al., Investigation of the Potential Use of Sialic Acid as a Biomarker for Rheumatoid Arthritis. Annals of Clinical and Laboratory Science, 2019. 49(2): p. 224-231.

14. Topcuoglu, C., et al., Total-and lipid-associated sialic acid in serum and thrombocytes in patients with chronic heart failure. Clin Biochem, 2010. 43(4-5): p. 447-9.

15. Kibos, A. and A. Guerchicoff, Susceptibility genes for coronary heart disease and myocardial infarction. Acute Card Care, 2011. 13(3): p. 136-42.

16. Ambrose, J.A., et al., Angiographic morphology and the pathogenesis of unstable angina pectoris. J Am Coll Cardiol, 1985. 5(3): p. 609-16.

17. Yu, X.H., et al., Biomarkers of unstable angina pectoris and yangxin decoction intervention: An exploratory metabonomics study of blood plasma. Medicine (Baltimore), 2017. 96(21): p. e6998.

18. Al-Karkhi, I.H., A.E. Ibrahim, and A.K. Yaseen, Levels of insulin, IL-6 and CRP in patients with unstable angina. Adv Clin Exp Med, 2013. 22(5): p. 655-8.

19. Smith, D.A., et al., Serum levels of the antiinflammatory cytokine interleukin-10 are decreased in patients with unstable angina. Circulation, 2001. 104(7): p. 746-9.

20. Sadeghi, M., et al., Serum interleukin-18 and extent of coronary artery disease in unstable angina. ARYA Atheroscler, 2018. 14(3): p. 122-127.

21. Hanson, V.A., et al., Plasma sialidase activity in acute myocardial infarction. Am Heart J, 1987. 114(1 Pt 1): p. 5963.

22. Haq, M., et al., Serum Total Sialic-Acid and Lipid-Associated Sialic-Acid in Normal Individuals and Patients with Myocardial-Infarction, and Their Relationship to Acute-Phase Proteins. Annals of Clinical Biochemistry, 1993. 30: p. 383-386.

23. Gokmen, S.S., et al., Association between serum total and lipid-bound sialic acid concentration and the severity of coronary atherosclerosis. Journal of Laboratory and Clinical Medicine, 2002. 140(2): p. 110-118.

\section{Tables}




\begin{tabular}{|c|c|c|c|c|c|}
\hline & Control & UA & AMI & Death & $P$ value \\
\hline \# of cases & 87 & 171 & 254 & 84 & - \\
\hline Age(years) & $61.26 \pm 11.97$ & $62.62 \pm 9.27$ & $60.15 \pm 11.64$ & $74.9 \pm 8.41$ & $\begin{array}{l}P_{3} \\
<0.0001\end{array}$ \\
\hline Male/Female & $47 / 40$ & $90 / 81$ & $136 / 118$ & $40 / 44$ & $P>0.05$ \\
\hline $\mathrm{BMI}\left(\mathrm{Kg} / \mathrm{m}^{2}\right)$ & $25.06 \pm 3.34$ & $25.90 \pm 3.92$ & $25.62 \pm 4.67$ & $25.23 \pm 3.83$ & $P>0.05$ \\
\hline Smoking (\%) & $7(12.43)$ & $64(37.43)$ & $82(32.28)$ & $25(29.76)$ & $P<0.05$ \\
\hline Alcoholism (\%) & 12(13.79) & $37(21.64)$ & $44(17.32)$ & $13(15.48)$ & $P>0.05$ \\
\hline Emergency (\%) & - & $13(7.60)$ & 154(60.63) & $50(59.52)$ & - \\
\hline $\mathrm{SBP}(\mathrm{mm} / \mathrm{Hg})$ & $125.40 \pm 17.51$ & $133.60 \pm 18.01$ & $128.10 \pm 20.05$ & $122.90 \pm 24.09$ & $P_{1}<0.001$ \\
\hline $\mathrm{DBP}(\mathrm{mm} / \mathrm{Hg})$ & $75.10 \pm 11.24$ & $75.72 \pm 12.44$ & $75.56 \pm 12.11$ & $70.06 \pm 12.28$ & $\begin{array}{l}P_{3} \\
<0.0001\end{array}$ \\
\hline \multirow[t]{3}{*}{ LVEF (\%) } & $64.74 \pm 5.87$ & $61.52 \pm 4.21$ & $57.07 \pm 7.56$ & $47.44 \pm 11.26$ & $P_{1}<0.001$ \\
\hline & & & & & $P_{2}<0.0001$ \\
\hline & & & & & $P_{3}<0.0001$ \\
\hline Hypertension (\%) & - & $109(63.74)$ & $131(51.57)$ & $50(59.52)$ & - \\
\hline Diabetes (\%) & - & $34(19.88)$ & $68(26.77)$ & $26(30.95)$ & - \\
\hline $\begin{array}{l}\text { Single/double vascular lesion } \\
(\%)\end{array}$ & - & $78(45.61)$ & 138(54.33) & $30(35.71)$ & - \\
\hline Triple vascular lesion (\%) & - & $48(28.07)$ & 101(39.76) & $22(26.19)$ & - \\
\hline $\begin{array}{l}\text { Cardiac function class at } \\
\text { admission }>\| 1(\%)\end{array}$ & - & $12(7.02)$ & $8(3.15)$ & $26(30.95)$ & - \\
\hline \multicolumn{6}{|l|}{ Concurrent medications } \\
\hline ACEI/ARB (\%) & - & $41(23.98)$ & $35(13.78)$ & 11(13.10) & - \\
\hline Beta-blockers (\%) & - & $17(9.94)$ & $50(19.69)$ & $8(9.52)$ & - \\
\hline CCB (\%) & - & $35(20.47)$ & $36(14.17)$ & $22(26.19)$ & - \\
\hline Diuretic (\%) & - & $9(5.26)$ & $10(3.94)$ & $3(3.57)$ & - \\
\hline Statins (\%) & - & $9(5.26)$ & $6(2.36)$ & $2(2.38)$ & - \\
\hline Aspirins (\%) & - & $16(9.36)$ & $8(3.15)$ & 0 & - \\
\hline Colpidogrel (\%) & - & $5(2.92)$ & $4(1.57)$ & 0 & - \\
\hline Insulin (\%) & - & $8(4.68)$ & $17(6.69)$ & $4(4.76)$ & - \\
\hline Hypoglycemic (\%) agents (\%) & - & $25(14.62)$ & $37(14.57)$ & $17(20.24)$ & - \\
\hline
\end{tabular}

Table 1. Clinical and angiographic characteristics of four groups: control, unstable angina (UA), acute myocardial infarction (AMI), death occurred within 24 hours of AMI in hospital (Death). BMI: body mass index, SBP: systolic blood 
pressure, DBP: diastolic blood pressure, $L V E F$ : left ventricular ejection fraction, AECl: angiotensin converting enzyme inhibitors, $A R B$ : angiotensin receptor blocker, CCB: calcium channel blocker. $P_{7}$ : control vs UA, $P_{2}$ : control vs $A M I, P_{3}$ : control vs Death, $P$ : refer to all of three groups. 


\begin{tabular}{|c|c|c|c|c|c|c|c|}
\hline & Control & UA & AMI & Death & $\mathrm{P}_{1}$ & $\mathrm{P}_{2}$ & $P_{3}$ \\
\hline \multirow[t]{2}{*}{$\mathrm{SA}(\mathrm{mg} / \mathrm{L})$} & 497.6 & 530.4 & 587.2 & 706.7 & \multirow[t]{2}{*}{$<0.001$} & \multirow[t]{2}{*}{$<0.0001$} & \multirow[t]{2}{*}{$<0.0001$} \\
\hline & $(458.2,527.7)$ & $(484.5,576.6)$ & $(527.6,671.6)$ & $(597.9,789.4)$ & & & \\
\hline \multirow{2}{*}{$\begin{array}{l}\mathrm{cTnl} \\
(\mathrm{ng} / \mathrm{mL})\end{array}$} & \multirow[t]{2}{*}{ - } & 0.004 & 1.943 & 2.730 & \multirow[t]{2}{*}{ - } & \multirow[t]{2}{*}{ - } & \multirow[t]{2}{*}{-} \\
\hline & & $(0.002,0.011)$ & $(0.204,12.840)$ & $(0.653,12.800)$ & & & \\
\hline \multirow{2}{*}{$\begin{array}{l}\text { Myoglobin } \\
\text { (ng/mL) }\end{array}$} & \multirow[t]{2}{*}{ - } & 30.7 & 50.4 & 174.1 & \multirow[t]{2}{*}{-} & \multirow[t]{2}{*}{-} & \multirow[t]{2}{*}{ - } \\
\hline & & $(24.4,40.5)$ & $(30.3,137.5)$ & $(67.9,372.9)$ & & & \\
\hline \multirow{2}{*}{$\begin{array}{l}\text { CKMB } \\
(\mathrm{ng} / \mathrm{mL})\end{array}$} & \multirow[t]{2}{*}{-} & 0.7 & 4.3 & 16.4 & \multirow[t]{2}{*}{-} & \multirow[t]{2}{*}{-} & \multirow[t]{2}{*}{ - } \\
\hline & & $(0.5,1.1)$ & $(1.4,34.3)$ & $(4.6,62.6)$ & & & \\
\hline \multirow{2}{*}{$\begin{array}{l}\mathrm{BNP} \\
(\mathrm{pg} / \mathrm{mL})\end{array}$} & \multirow[t]{2}{*}{ - } & 35 & 117 & 1240 & \multirow[t]{2}{*}{ - } & \multirow[t]{2}{*}{ - } & \multirow[t]{2}{*}{ - } \\
\hline & & $(18,75)$ & $(50,259)$ & $(406,3850)$ & & & \\
\hline \multirow{2}{*}{$\begin{array}{l}\text { LDL } \\
(\mathrm{mmol} / \mathrm{L})\end{array}$} & 2.58 & 2.38 & 2.49 & 1.98 & \multirow[t]{2}{*}{$>0.05$} & \multirow[t]{2}{*}{$>0.05$} & \multirow[t]{2}{*}{$<0.05$} \\
\hline & $(1.93,3.11)$ & $(1.76,2.89)$ & $(2.09,3.05)$ & $(1.62,2.70)$ & & & \\
\hline \multirow{2}{*}{$\begin{array}{l}\text { TG } \\
(\mathrm{mmol} / \mathrm{L})\end{array}$} & 1.05 & 1.43 & 1.40 & 1.08 & $<0.001$ & $<0.0001$ & $>0.05$ \\
\hline & $(0.80,1.53)$ & $(0.99,2.20)$ & $(1.01,2.23)$ & $(0.81,1.51)$ & & & \\
\hline $\mathrm{HDL}$ & 1.32 & 1.09 & 1.10 & 1.09 & $<0.0001$ & $<0.0001$ & $<0.0001$ \\
\hline & $(1.15,1.59)$ & $(0.97,1.31)$ & $(0.94,1.27)$ & $(0.85,1.28)$ & & & \\
\hline FFA & 0.41 & 0.50 & 0.70 & 0.74 & $>0.05$ & $<0.0001$ & $<0.0001$ \\
\hline & $(0.30,0.67)$ & $(0.31,0.75)$ & $(0.48,0.96)$ & $(0.56,1.03)$ & & & \\
\hline ApoA1 & 1.18 & 1.27 & 1.22 & 1.01 & $>0.05$ & $>0.05$ & $>0.05$ \\
\hline & $(1.09,1.38)$ & $(1.05,1.47)$ & $(1.03,1.43)$ & & & & \\
\hline ApoB & 0.79 & 0.84 & 0.88 & 0.79 & $>0.05$ & $>0.05$ & $>0.05$ \\
\hline & $(0.59,0.94)$ & $(0.68,1.00)$ & $(0.70,1.03)$ & $(0.65,1.00)$ & & & \\
\hline $\operatorname{Lp}(a)$ & 98 & 153 & 193 & 212 & $<0.05$ & $<0.0001$ & $<0.0001$ \\
\hline & $(52,200)$ & $(101,245)$ & $(115,293)$ & $(122,348)$ & & & \\
\hline TC & 4.46 & 4.08 & 4.00 & 3.68 & $>0.05$ & $<0.001$ & $<0.001$ \\
\hline & $(3.71,5.29)$ & $(3.31,4.80)$ & $(2.99,4.80)$ & $(3.12,4.61)$ & & & \\
\hline GLU & 5.22 & 4.86 & 5.32 & 6.50 & $>0.05$ & $>0.05$ & $<0.001$ \\
\hline 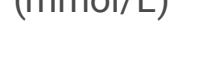 & $(4.50,5.96)$ & $(4.44,5.54)$ & $(4.77,6.99)$ & $(5.01,9.79)$ & & & \\
\hline $\operatorname{ADA}(\mathrm{U} / \mathrm{L})$ & 9 & 10 & 11 & 10 & $<0.001$ & $<0.0001$ & $<0.01$ \\
\hline & $(7,11)$ & $(9,13)$ & $(9,13)$ & $(9,13)$ & & & \\
\hline AFU (U/L) & 27 & 24 & 24 & 24 & $<0.001$ & $<0.01$ & $<0.01$ \\
\hline & $(24,32)$ & $(19,28)$ & $(21,28)$ & $(19,30)$ & & & \\
\hline
\end{tabular}




\begin{tabular}{|llllllll|} 
LDH (U/L) & 157 & 163 & 270 & 396 & $>0.05$ & $<0.0001$ & $<0.0001$ \\
& $(143,177)$ & $(143,184)$ & $(187,416)$ & $(256,683)$ & & & \\
\hline TP $(\mathrm{g} / \mathrm{L})$ & 67.5 & 63.3 & 64.7 & 59.1 & $<0.0001$ & $>0.05$ & $<0.0001$ \\
& $(62.9,71.5)$ & $(59.6,67.0)$ & $(59.5,75.9)$ & $(55.6,62.4)$ & & & \\
\multirow{2}{*}{ A/G } & 1.7 & 1.6 & 1.5 & 1.3 & $>0.05$ & $<0.01$ & $<0.0001$ \\
& $(1.46,1.90)$ & $(1.40,1.70)$ & $(1.40,1.80)$ & $(1.02,1.53)$ & & & \\
\hline \multirow{2}{*}{ GS } & - & 20.5 & 47.5 & 55.0 & - & - & - \\
& & $(5.0,51.8)$ & $(28.3,74.0)$ & $(42.5,100.0)$ & & & \\
\hline
\end{tabular}

Table 2. The laboratory parameters and Gensini Score of four groups. $P_{1}$ stands for $P$ value of UA and control. $P_{2}$ stands for $P$ value of $A M I$ and control. $P_{3}$ stands for $P$ value of Death and control. SA: sialic acid, $c T$ Tnl: cardiac troponin I, CKMB: creatine kinase isoenzyme, BNP: type B natriuretic peptide, $L D L$ : low density lipoprotein, TG: triglyceride, $H D L$ : high density lipoprotein, FFA: free fatty acid, ApoA1: apolipoprotein A1, ApoB: apolipoprotein B, Lp(a): lipoprotein a, TC: total cholesterol, GLU: glucose, ADA: adenosine deaminase, AFU: fucosidase, LDH: lactic dehydrogenase, TP: total protein, A/G: albumin/globulin, GS: gensini score.

The univariate regression analysis of unstable angina

\begin{tabular}{|llll|}
\hline Variable & OR & $95 \% \mathrm{Cl}$ & $\mathrm{P}$ \\
\hline SA & 1.010 & $(1.005,1.015)$ & 0.000 \\
HDL & 0.085 & $(0.032,0.225)$ & 0.000 \\
TG & 1.495 & $(1.084,2.060)$ & 0.014 \\
LVEF & 0.000 & $(0.000,0.0000)$ & 0.000 \\
\hline
\end{tabular}

The multivariate regression analysis of unstable angina.

\begin{tabular}{|llll|}
\hline Variable & OR & $95 \% \mathrm{Cl}$ & $\mathrm{P}$ \\
\hline $\mathrm{SA}$ & 1.011 & $(1.006,1.016)$ & 0.000 \\
\hline $\mathrm{HDL}$ & 0.253 & $(0.071,0.903)$ & 0.034 \\
\hline
\end{tabular}

Table 3. The univariate and multivariate regression analysis of unstable angina. OR: odds ratio; Cl: confidential interval. SA: sialic acid, HDL: high density lipoprotein, TG: triglyceride, LVEF: left ventricular ejection fraction.

The univariate regression analysis between AMI and Death. 


\begin{tabular}{|llll|}
\hline Variable & OR & $95 \% \mathrm{Cl}$ & $\mathrm{P}$ \\
\hline BNP & 1.001 & $(1.001,1.002)$ & 0.000 \\
\hline SA & 1.006 & $(1.002,1.010)$ & 0.001 \\
apoA1 & 0.048 & $(0.004,0.512)$ & 0.012 \\
\hline LVEF & 0.000 & $(0.000,0.018)$ & 0.000 \\
\hline Glu & 1.165 & $(1.005,1.349)$ & 0.043 \\
\hline GS & 1.022 & $(1.008,1.036)$ & 0.002 \\
age & 1.141 & $(1.071,1.215)$ & 0.000 \\
\hline
\end{tabular}

The multivariate regression analysis between AMI and Death.

\begin{tabular}{|llll|}
\hline Variable & OR & $95 \% \mathrm{Cl}$ & $\mathrm{P}$ \\
\hline BNP & 1.001 & $(1.000,1.002)$ & 0.009 \\
\hline age & 1.141 & $(1.046,1.245)$ & 0.003 \\
\hline
\end{tabular}

Table 4. The univariate and multivariate regression analysis between AMI and Death. OR: odds ratio; Cl: confidential interval. BNP: type B natriuretic peptide, SA: sialic acid, apoA1: apolipoprotein A1, LVEF: left ventricular ejection fraction, Glu: glucose, GS: gensini score.

\begin{tabular}{|llll|}
\hline & Single/Double vascular lesion & Triple vascular lesion & $\mathrm{P}$ value \\
\hline \# of cases & 138 & 101 & - \\
\hline Age(years) & $58.33 \pm 11.00$ & $62.46 \pm 12.17$ & $\mathrm{P}<0.05$ \\
\hline Male/Female & $79 / 59$ & $54 / 47$ & $\mathrm{P}>0.05$ \\
\hline BMl(Kg/m2) & $26.02 \pm 3.51$ & $25.37 \pm 4.02$ & $\mathrm{P}>0.05$ \\
\hline Smoking (\%) & $49(35.51)$ & $32(31.68)$ & $\mathrm{P}>0.05$ \\
\hline Alcoholism (\%) & $24(17.39)$ & $18(17.82)$ & $\mathrm{P}>0.05$ \\
\hline Emergency (\%) & $86(62.32)$ & $59(58.42)$ & $\mathrm{P}>0.05$ \\
\hline SBP (mm/Hg) & $126 \pm 18$ & $130 \pm 22$ & $\mathrm{P}>0.05$ \\
\hline DBP (mm/Hg) & $75 \pm 12$ & $76 \pm 12$ & $\mathrm{P}>0.05$ \\
\hline LVEF $(\%)$ & $57.49 \pm 7.20$ & $56.59 \pm 7.52$ & $\mathrm{P}>0.05$ \\
\hline Hypertension (\%) & $60(43.48)$ & $59(58.42)$ & $\mathrm{P}<0.05$ \\
\hline Diabetes (\%) & $30(21.74)$ & $35(34.65)$ & $\mathrm{P}<0.05$ \\
\hline
\end{tabular}

Table 5. The baseline information of groups of various number of lesion vessels.

BMI: body mass index, SBP: systolic blood pressure, DBP: diastolic blood pressure,

LVEF:left ventricular ejection fraction 


\section{Figures}



Figure 1

Heat map of laboratory parameters among four groups. The levels of various parameters in control group were considered as " 1 ". The ratio of other three groups to control group were calculated and used to draw the heat map. When the serum biomarker levels were lower than healthy controls, blue color was used in the plot whereas when the serum biomarker levels were higher than healthy controls, red color was used.

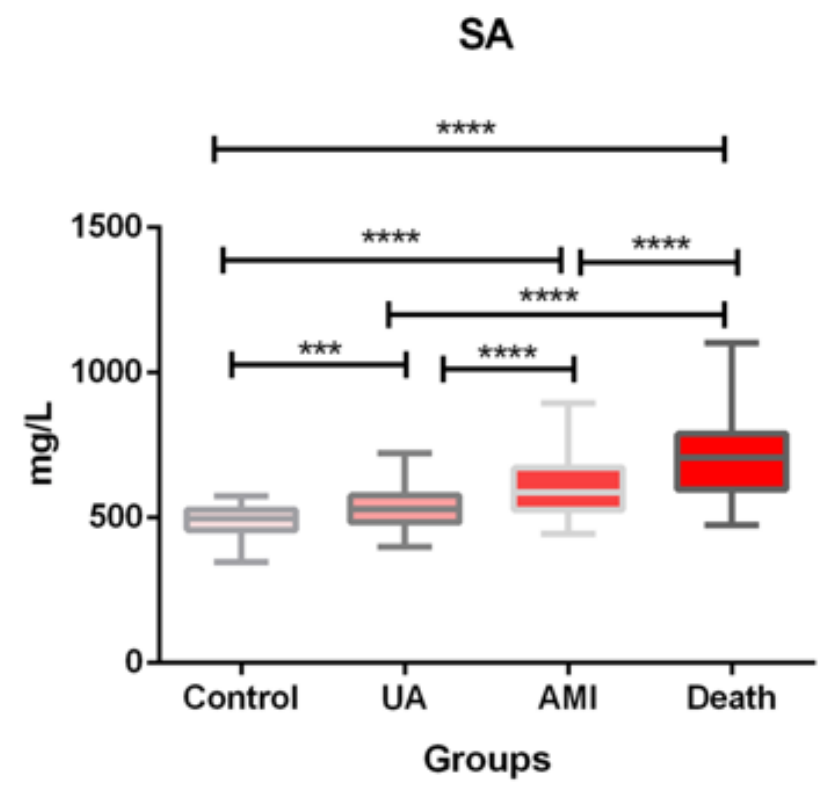

Figure 2 
. Serum SA levels in the validation population. The box plots show the expression levels of serum SA in unstable angina (UA), acute myocardial infarction (AMI), death post-AMI (Death) and control group. ${ }^{\star \star *} \mathrm{P}<0.001,{ }^{\star \star \star *} \mathrm{P}<0.0001$. A 95\% confidence interval is maintained.

a

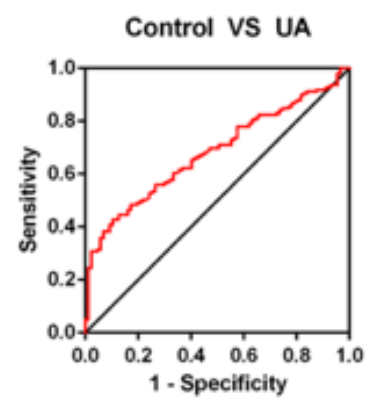

d

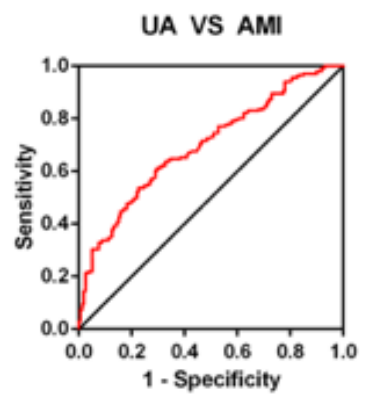

b



e

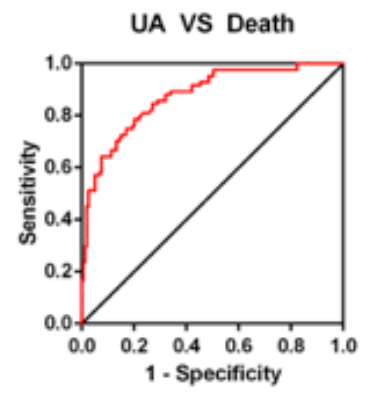

C



f



\begin{tabular}{cccccc}
\hline & cut off & Sensitivity & Specificity & AUC & P value \\
\hline Control VS UA & $>542.8$ & 0.4277 & 0.8966 & 0.6815 & $<0.0001$ \\
Control VS AMl & $>560.4$ & 0.6169 & 0.9770 & 0.8419 & $<0.0001$ \\
Control VS Death & $>553.8$ & 0.8929 & 0.9425 & 0.9639 & $<0.0001$ \\
UA VS AMI & $>561.9$ & 0.6119 & 0.7044 & 0.6968 & $<0.0001$ \\
UA VS Death & $>591.3$ & 0.7857 & 0.7987 & 0.8754 & $<0.0001$ \\
AMI VS Death & $>638.9$ & 0.6667 & 0.6617 & 0.7192 & $<0.0001$ \\
\hline
\end{tabular}

\section{Figure 3}

The relative operating characteristic curve (ROC curve) of serum SA between any two groups. Sensitivity, specificity and area under the curve (AUC) of ROC curve were concluded. 


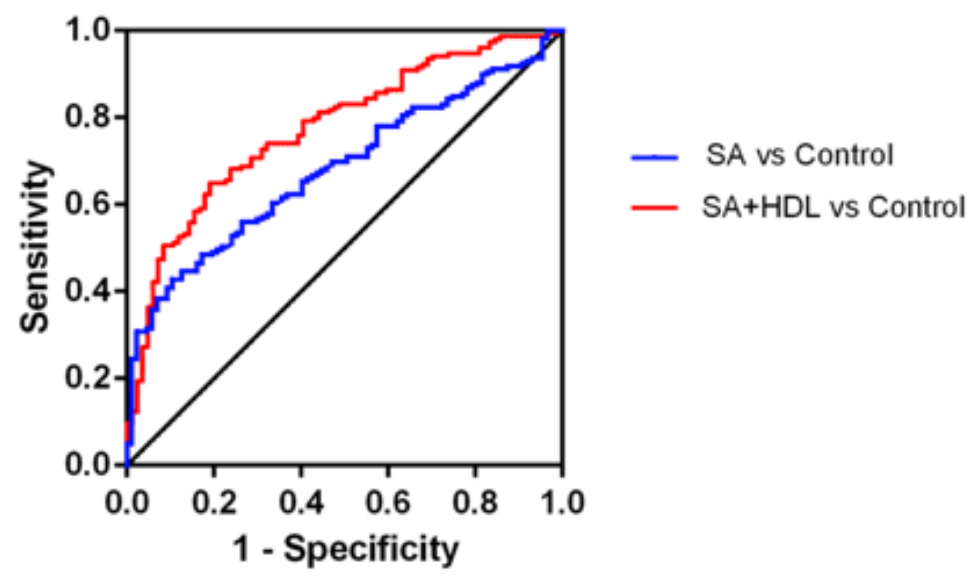

\begin{tabular}{ccccc}
\hline & Sensitivity & Specificity & AUC & P value \\
\hline SA+HDL & 0.6494 & 0.8095 & 0.7754 & $<0.0001$ \\
SA & 0.4277 & 0.8966 & 0.6815 & $<0.0001$ \\
\hline
\end{tabular}

Figure 4

Comparison of ROC curve of serum SA and serum SA as well as HDL between control and UA groups.

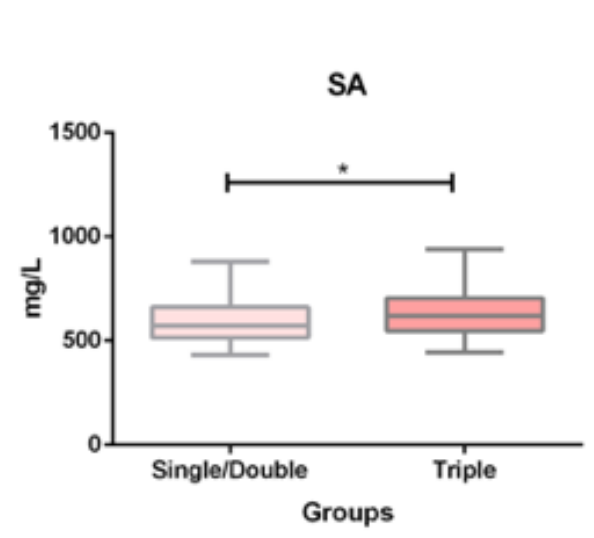

A

\section{Figure 5}

Serum SA levels in the Single/Double vascular lesion group and Triple vascular lesion group (A). * $<<0.05$. A 95\% confidence interval is maintained. The relative operating characteristic curve (ROC curve) of serum SA between any two groups (B). Sensitivity, specificity and area under the curve (AUC) of ROC curve were concluded. 